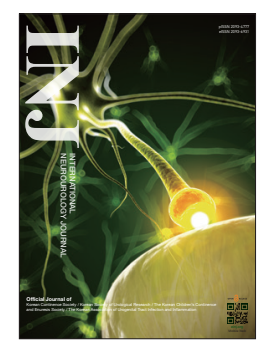

\title{
Urine Proteomics in the Era of Mass Spectrometry
}

\author{
Ashley Beasley-Green \\ Material Measurement Laboratory, Biomolecular Measurement Division, National Institute of Standards and Technology, Gaithersburg, MD, USA
}

With the technological advances of mass spectrometry (MS)-based platforms, clinical proteomics is one of the most rapidly growing areas in biomedical research. Urine proteomics has become a popular subdiscipline of clinical proteomics because it is an ideal source for the discovery of noninvasive disease biomarkers. The urine proteome offers a comprehensive view of the local and systemic physiology since the proteome is primarily composed of proteins/peptides from the kidneys and plasma. The emergence of MS-based proteomic platforms as prominent bioanalytical tools in clinical applications has enhanced the identification of protein-based urinary biomarkers. This review highlights the characteristics of urine that make it an attractive biofluid for biomarker discovery and the impact of MS-based technologies on the clinical assessment of urinary protein biomarkers.

Keywords: Urine; Biomarkers; Proteomics; Mass Spectrometry

- NIST Disclaimer: Certain commercial equipment, instruments, and materials are identified in this paper to adequately specify the experimental procedure. Such identifications do not imply recommendations or endorsement by National Institute of Standards and Technology (NIST) nor do they imply that the equipment, instruments, or materials are necessarily the best available for the purpose.

- Conflict of Interest: No potential conflict of interest relevant to this article was reported.

\section{INTRODUCTION}

The goal of this article is to provide a summary of the advancement of urine proteomics in clinical applications with the emergence of sophisticated mass spectrometry (MS)-based proteomic platforms. Urine is one of the most valuable biofluids for biomarker discovery because it is available in almost all patients at relatively large quantities and the collection of urine is simple and noninvasive compared to other biofluids. The kidneys produce urine via the process of ultrafiltration and urine is primarily used to eliminate soluble waste products, such as electrolytes, nitrogenous compounds and hormones, from plasma [1]. The glomerulus filters roughly 150-180 L of plasma to develop the "primitive urine," more than 99\% of "primitive urine" is reabsorbed by the renal tubule and the "final urine" is excreted through the urethra [1]. Normal excretion of protein in urine is less than $150 \mathrm{mg} / \mathrm{L}$ per 24 hours and approximately $30 \%$ of urinary protein originates from plasma via blood filtration, whereas the remaining $70 \%$ is derived from the kidneys [2].

Proteomics is an important postgenomic approach that can provide a qualitative and quantitative assessment of the proteome of biological systems [3]. Characterizing a proteome can offer clues about the pathological and physiological processes in a biological system and provide critical insight on the development and progression of disease [4]. Therefore, analysis of the urine proteome could be extremely useful in the characterization of pathophysiological mechanisms and the identifica-
Corresponding author: Ashley Beasley-Green (iD http://orcid.org/0000-0002-2065-4218 Material Measurement Laboratory, Biomolecular Measurement Division, National Institute of Standards and Technology, 100 Bureau Drive, MS 8314, Gaithersburg, MD 20899-8390, USA

E-mail: ashley.beasley@nist.gov / Tel: +1-301-975-4026 / Fax: +1-301-977-0685 Submitted: September 23, 2016 / Accepted after revision: October 6, 2016
This is an Open Access article distributed under the terms of the Creative Commons Attribution Non-Commercial License (http://creativecommons.org/licenses/by-nc/4.0/) which permits unrestricted non-commercial use, distribution, and reproduction in any medium, provided the original work is properly cited. 
tion of therapeutic targets of kidney and non-kidney diseases. Various proteomic-based technologies have been utilized to study the urine proteome; however, MS-based platforms have emerged as one of the most suitable platforms for urinary protein and peptide profiling in clinical applications. This short review will highlight the following aspects of urine proteomics: the proteomic studies directed toward the complete characterization of normal urine proteome; the advances in MS-based approaches in urinary proteomics; and clinical applications of MS-based urine proteomics to assess kidney disease.

\section{NORMAL HUMAN URINARY PROTEOME}

Normal human urine contains a significant amount of proteins and peptides and over the past decade there has been extensive profiling of the normal urine proteome. In the past, numerous studies utilized 2-dimensional electrophoresis (2-DE) to characterize urinary proteins; however, Spahr et al. [5] in $2001 \mathrm{em}$ ployed liquid chromatography-tandem mass spectrometry (LC-MS/MS) in an attempt to profile unfractionated urine proteome. From this initial study, a total of 124 proteins were identified, with the majority of the MS spectra originating from nine of the most abundant proteins observed in plasma, including: human serum albumin (HSA), serotransferrin, Ig kappa light chain c, Ig gamma heavy chain c, uromodulin, apolipoprotein a-i, alpha-1 microglobulin, zinc-alpha-2 glycoprotein, and alpha-1-antitrypsin [5]. In 2004, this number increased to 150 distinct proteins utilizing size-exclusion chromatographic fractionation and 2-DE separation followed by LC-MS/MS analysis [6]. This number increased significantly in 2006 to 1,543 proteins by combining 1 -dimensional gel electrophoresis and liquid chromatography coupled to high-resolution mass spectrometric instrumentation (LTQ-FT, LTQ-Orbitrap) from normal single donor and pooled urine [7]. To date, over 2,000 proteins have been identified in normal human urine, with 1,823 being identified by Marimuthu et al. [8] in 2011 via both a nonaffinity and lectin affinity-based approach followed by sodium dodecyl sulfate polyacrylamide gel electrophoresis separation and high-resolution LC-MS/MS analysis (LTQ-Orbitrap). In addition to the vast number of proteins in normal urine, the protein/peptide composition is greatly influenced by various factors, such as sex, age, diet, lifestyle, and physiological condition, and can change significantly over time. This variation in protein/peptide composition further complicates the characterization of normal or diseased urine proteome and subsequently the identification of protein/peptide biomarkers in urine.

\section{MS-BASED TECHNIQUES FOR URINE PROTEOMIC STUDIES}

Over the years various MS technologies have been developed with varying degrees of analytical performance in terms of mass resolution, reproducibility, selectivity, and sensitivity. Of these technologies, multiple MS-based platforms have been employed to characterize the urine proteome in both an untargeted (global profiling) and targeted approach. A common experimental strategy used in urine proteomic experiments to reduce the complexity of the proteome is the "divide and conquer" strategy, which involves protein separation, fractionation or enrichment followed by protein identification via MS [9]. MS technologies used in urine proteome investigations include the following: matrix-assisted laser desorption/ionization-timeof-flight (MALDI-TOF) MS, high-resolution MS and triple quadrupole MS.

\section{MALDI-TOF MS}

MALDI-TOF instrumentation can be used in both a targeted and untargeted proteomic approach. In MALDI-TOF MS, the nonvolatile analyte is mixed on a stainless steel target plate with excess matrix, a small ultraviolet-absorbing aromatic organic compound (i.e., sinapinic acid, 2,5-dihydroxybenzoic acid, or a-cyano-4-hydroxycinnamic acid), to promote ionization and vaporization of the analyte. The matrix absorbs energy from a laser beam and ionization of the analyte occurs via proton transfer between the matrix and the analyte, which is referred to as the translational stage. The charged analyte species are subjected to a field-free drift tube (TOF mass analyzer) where they are separated on the basis of their mass-to-charge ratio $(\mathrm{m} / \mathrm{z})$. Utilizing MALDI-TOF MS for the identification and characterization of urinary proteins/peptides poses several advantages including: reduced analysis time; enhanced detection sensitivity; extensive mass range; simple mass spectrum due to the production of singly-charged species; enhanced mass resolution when equipped with a reflectron; increased tolerance for contaminants; and molecular imaging capabilities. Although MALDI-TOF MS is an ideal technique for simple matrices, analysis of urine requires significant sample preparation techniques, including protein fractionation or enrichment prior to MS analysis, to prevent un- 
dersampling of the urine proteome and suppression of low abundant proteins. Previous studies have employed MALDI-TOF MS to characterize the normal urine proteome [6].

\section{High-Resolution MS}

In most urine proteomic studies, the high-resolution mass analyzers are coupled to a linear ion trap (i.e., LTQ), including the LTQ-FT (Fourier-transform, FT) and the LTQ-Orbitrap. A Fourier-transform mass spectrometer (FT-MS) is a superconducting magnet-based analyzer that provides high mass accuracy (sub-parts-per-million mass accuracy), dynamic range and resolving power, which has been shown to increase the number of high-confidence protein identifications [10]. A major disadvantage of FT-MS instruments is their relatively slow acquisition rates, which could result in low sampling of complex samples, such as urine. In addition to the potential for undersampling, the complexity of the superconducting magnet is also a drawback to the FT-MS instrumentation. Therefore, the Orbitrap analyzer offers a comparable alternative due to the fact that it is a magnetic-field free mass analyzer [10] and the LTQOrbitrap instrument has been utilized in numerous untargeted urine proteomic studies [7]. In addition to mass accuracy, the LTQ-Orbitrap platform also supports additional fragmentation methods, including electron transfer dissociation $[11,12]$ and higher-energy collision dissociation [13], which expands the number of possible protein/peptide identifications in urine. The LTQ-Orbitrap mass spectrometer is used in an untargeted (global profiling) approach to identify proteins in urine; however, with advances in the technology, the system can also be used in a targeted approach [14-16].

\section{Triple Quadrupole MS}

Quantitative proteomics has emerged as a premier bioanalytical approach in routine biomedical research and clinical experimental workflows and the triple quadrupole mass spectrometer is the instrument of choice. The triple quadrupole mass spectrometer is composed of 3 quadrupoles, the first and third quadrupole function as mass filters to select predefined $\mathrm{m} / \mathrm{z}$ values corresponding to the peptide precursor ion and the fragment (product) ion of the peptide, respectively [17]. The second quadrupole operates as a collision cell for collision-induced dissociation of the precursor ion to generate the product ions [17]. The precursor ion/product ion pair represents a multiple reaction monitoring (MRM) transition. Due to the high specificity of this approach, multiple peptides (proteins) can be targeted in a single experiment which is the basis for multiplexed MSbased protein assays. In this MS-based quantitative approach, the high degree of selectivity and sensitivity of the MRM mode that the triple quadrupole MS offers coupled with the incorporation of stable isotopically labeled internal standards enables the targeted absolute quantification of analytes in highly complex biological matrices.

\section{CLINICAL APPLICATIONS OF URINARY PROTEOMICS}

Characterization of the urine proteome can be useful in the generation of a comprehensive view of the pathophysiological mechanisms in the kidneys and the potential discovery of biomarkers and therapeutic targets of kidney and non-kidney diseases. Urine albumin characterization and quantification provides an example of how MS-based proteomic platforms can be effective in clinical applications [18].

\section{Urine Albumin}

Urine albumin is a major biomarker for early diagnosis, prognosis, and disease management of renal disease and is critical for clinical decisions associated with renal therapy. Therefore, precise measurement of urine albumin plays an important role in the early detection of renal dysfunction, evaluation of treatment efficacy, and reduction in the risk of kidney failure and cardiovascular disease [19]. To selectively measure albumin in urine, current clinical methodologies utilize affinity-based techniques such as urine albumin-based enzyme-linked immunosorbent assays, immunoturbidity assays, and radioimmunoassays $[20,21]$. Although affinity-based assays are routinely used in clinical laboratories to assess patient samples, there are distinct measurement challenges for the current methods, with the leading issue being heterogeneity of albumin species in urine. The presence of modified (i.e., glycated albumin) [22-24] or fragmented (i.e., N- and C-terminal truncation products) [2529] forms of albumin in urine can alter the antibody recognition site for routine clinical immunoassays and thus potentially bias the total urine albumin measurement [22-29]. Moreover, variations in urine albumin can also affect the analytical precision and intra- and interlaboratory measurement comparability of commonly used affinity-based assays [19]. Therefore, due to the clinical importance of the urine albumin measurement, the National Institute of Standards and Technology (NIST) has been assisting the National Kidney Disease Education Program 
and the Working Group for the Standardization of Albumin Assays in Urine of the International Federation of Clinical Chemistry in the development of a reference measurement system for urine albumin to support measurement accuracy and comparability across laboratories [30,31]. In support of this effort, NIST has developed a multiplexed candidate reference measurement procedure that utilizes isotope dilution-mass spectrometry and MRM to quantify full-length urine albumin [18]. The multiplexed MS assay incorporates an isotopically-labeled $\left({ }^{15} \mathrm{~N}\right)$ full-length recombinant HSA material as the internal standard, which permits the absolute quantitation of fulllength albumin in human urine [18]. Incorporation of the fulllength internal standard offers the following advantages for a multiplexed quantification assay: (1) The ability to target multiple peptides, irrespective of amino acid composition, that span the full amino acid sequence of the analyte; (2) The ability to account for preanalytical bias (i.e., proteolytic digestion efficiency and analyte recovery) due to the incorporation of the internal standard during the initial sample processing phase; and (3) The ability to quantitatively and qualitatively (analyte structure analysis) assess the target analyte in a single experiment. The LTQ-Orbitrap mass spectrometer was employed to identify target peptides that span the HSA amino acid sequence and the triple quadrupole mass spectrometer (MRM mode) was used to quantify urine albumin based on the selected peptides [18]. In addition to the quantitative advantages, use of multiple peptides that span the HSA sequence enables the qualitative assessment of molecular heterogeneity of endogenous urine albumin. Therefore, introducing MS-based platforms into the proteomic workflow for urine albumin assessment can enhance the accuracy and precision of urine albumin measurements and ultimately facilitate early diagnosis of kidney dysfunction.

\section{CHALLENGES IN MS-BASED URINE PROTEOMIC STUDIES}

A major challenge researchers face when utilizing MS-based platforms to investigate urine is proteome variability, which is a common theme in most proteomic studies of biological fluids. In urine, proteome variability is associated with a number of factors, including inter- and intrapatient biological variability, diet, age, sex, and disease state. Due to the nature of urine, being the biofluid for excretion, the protein/peptide concentration and composition is highly dynamic and varies within and between individuals, which reduces the reproducibility of protein mea- surements in urine. Therefore, protein heterogeneity negatively influences the precision and accuracy of MS-based measurement of urine, which could hinder the discovery of stable and specific urinary biomarkers. In addition to protein/peptide heterogeneity, the low concentration of protein present in normal urine coupled with the presence of urea, salts and other components could interfere with protein identification by MS through signal suppression and undersampling. The dynamic range of urinary proteins can alter the characterization of low abundant proteins excreted in normal or diseased urine. Therefore, extensive sample preparation techniques, such as desalting, enrichment, fractionation, and separation, should be considered in urinary proteomic investigations to reduce the complexity of the proteome. Reducing the complexity of the urine proteome and removal of signal suppressive agents, such as urea and salts, could broaden the scope of the proteome and facilitate the identification of both high and low abundant proteins. In addition to urine-specific characteristics, there are also challenges associated with MSbased platforms that can influence MS measurement quality. In routine proteomic workflows, the proteins undergo proteolytic digestion to produce peptides prior to MS assessment. Suboptimal digestion conditions could affect the digestion efficiency of the proteolytic enzyme and bias the MS measurements. This bias could prevent the comprehensive profile of the proteome. Overall, there are several factors associated with sample preparation (such as fractionation, enrichment, and proteolysis) and sample processing (such as ion suppression, detection specificity, and instrument sensitivity) techniques that can influence the quality of MS-based measurements of the urine proteome. Therefore, the proteomic workflow should be optimized for urine analysis to reduce proteome complexity and enable the characterization of both high and low abundant proteins.

\section{CONCLUSION}

Urine is a valuable biofluid for the discovery of noninvasive biomarkers and the proteome offers a comprehensive view of the local and systemic physiology. The incorporation of MSbased technologies in urinary proteomic experimental workflows has broadened the understanding of normal and diseaserelated mechanisms in the kidney. Moreover, usage of MS instrumentation in urine-based clinical diagnostics will enable the early detection of renal dysfunction, provide an in-depth assessment of treatment efficacy, and potentially reduce the risk of kidney failure. 


\section{REFERENCES}

1. Decramer S, Gonzalez de Peredo A, Breuil B, Mischak H, Monsarrat B, Bascands JL, et al. Urine in clinical proteomics. Mol Cell Proteomics 2008;7:1850-62.

2. Thongboonkerd V, Malasit P. Renal and urinary proteomics: current applications and challenges. Proteomics 2005;5:1033-42.

3. Azarkan M, Huet J, Baeyens-Volant D, Looze Y, Vandenbussche G. Affinity chromatography: a useful tool in proteomics studies. J Chromatogr B Analyt Technol Biomed Life Sci 2007;849:81-90.

4. Pierce JD, Fakhari M, Works KV, Pierce JT, Clancy RL. Understanding proteomics. Nurs Health Sci 2007;9:54-60.

5. Spahr CS, Davis MT, McGinley MD, Robinson JH, Bures EJ, Beierle J, et al. Towards defining the urinary proteome using liquid chromatography-tandem mass spectrometry. I. Profiling an unfractionated tryptic digest. Proteomics 2001;1:93-107.

6. Pieper R, Gatlin CL, McGrath AM, Makusky AJ, Mondal M, Seonarain $\mathrm{M}$, et al. Characterization of the human urinary proteome: a method for high-resolution display of urinary proteins on two-dimensional electrophoresis gels with a yield of nearly 1400 distinct protein spots. Proteomics 2004;4:1159-74.

7. Adachi J, Kumar C, Zhang Y, Olsen JV, Mann M. The human urinary proteome contains more than 1500 proteins, including a large proportion of membrane proteins. Genome Biol 2006;7:R80.

8. Marimuthu A, O'Meally RN, Chaerkady R, Subbannayya Y, Nanjappa V, Kumar P, et al. A comprehensive map of the human urinary proteome. J Proteome Res 2011;10:2734-43.

9. Beasley-Green A, Bunk D, Rudnick P, Kilpatrick L, Phinney K. A proteomics performance standard to support measurement quality in proteomics. Proteomics 2012;12:923-31.

10. Makarov A, Denisov E, Kholomeev A, Balschun W, Lange O, Strupat $\mathrm{K}$, et al. Performance evaluation of a hybrid linear ion trap/orbitrap mass spectrometer. Anal Chem 2006;78:2113-20.

11. Kim MS, Pandey A. Electron transfer dissociation mass spectrometry in proteomics. Proteomics 2012;12:530-42.

12. Dai S, Ni W, Patananan AN, Clarke SG, Karger BL, Zhou ZS. Integrated proteomic analysis of major isoaspartyl-containing proteins in the urine of wild type and protein L-isoaspartate O-methyltransferase-deficient mice. Anal Chem 2013;85:2423-30.

13. Pejchinovski M, Klein J, Ramírez-Torres A, Bitsika V, Mermelekas $\mathrm{G}$, Vlahou A, et al. Comparison of higher energy collisional dissociation and collision-induced dissociation MS/MS sequencing methods for identification of naturally occurring peptides in human urine. Proteomics Clin Appl 2015;9:531-42.

14. Lesur A, Domon B. Advances in high-resolution accurate mass spectrometry application to targeted proteomics. Proteomics 2015; 15:880-90.

15. Gallien S, Duriez E, Crone C, Kellmann M, Moehring T, Domon B. Targeted proteomic quantification on quadrupole-orbitrap mass spectrometer. Mol Cell Proteomics 2012;11:1709-23.

16. Khristenko N, Domon B. Quantification of proteins in urine samples using targeted mass spectrometry methods. Methods Mol Biol 2015;1243:207-20.

17. Lange V, Picotti P, Domon B, Aebersold R. Selected reaction monitoring for quantitative proteomics: a tutorial. Mol Syst Biol 2008; 4:222.

18. Beasley-Green A, Burris NM, Bunk DM, Phinney KW. Multiplexed LC-MS/MS assay for urine albumin. J Proteome Res 2014; 13:3930-9.

19. Kestenbaum B, de Boer IH. Urine albumin-to-creatinine ratio: what's in a number? J Am Soc Nephrol 2010;21:1243-4.

20. Choi S, Choi EY, Kim HS, Oh SW. On-site quantification of human urinary albumin by a fluorescence immunoassay. Clin Chem 2004; 50:1052-5.

21. Seegmiller JC, Sviridov D, Larson TS, Borland TM, Hortin GL, Lieske JC. Comparison of urinary albumin quantification by immunoturbidimetry, competitive immunoassay, and protein-cleavage liquid chromatography-tandem mass spectrometry. Clin Chem 2009;55:1991-4.

22. Dozio E, Di Gaetano N, Findeisen P, Corsi Romanelli MM. Glycated albumin: from biochemistry and laboratory medicine to clinical practice. Endocrine 2016 Sep 13 [Epub]. http://doi.org/10.1007/ s12020-016-1091-6.

23. Barnaby OS, Cerny RL, Clarke W, Hage DS. Quantitative analysis of glycation patterns in human serum albumin using 160/180-labeling and MALDI-TOF MS. Clin Chim Acta 2011;412:1606-15.

24. Cha T, Tahara Y, Yamato E, Yoneda H, Ikegami H, Noma Y, et al. Renal handling of glycated albumin in non-insulin-dependent diabetes mellitus with nephropathy. Diabetes Res Clin Pract 1991; 12:149-56.

25. Sviridov D, Drake SK, Hortin GL. Reactivity of urinary albumin (microalbumin) assays with fragmented or modified albumin. Clin Chem 2008;54:61-8.

26. Brennan SO, George PM. Three truncated forms of serum albumin associated with pancreatic pseudocyst. Biochim Biophys Acta 2000;1481:337-43.

27. Bar-Or D, Rael LT, Bar-Or R, Slone DS, Craun ML. The formation and rapid clearance of a truncated albumin species in a critically ill patient. Clin Chim Acta 2006;365:346-9.

28. Wiggins RC, Kshrisagar B, Kelsch RC, Wilson BS. Fragmentation 
and polymeric complexes of albumin in human urine. Clin Chim Acta 1985;149:155-63.

29. Candiano G, Musante L, Bruschi M, Petretto A, Santucci L, Del Boccio P, et al. Repetitive fragmentation products of albumin and alpha1-antitrypsin in glomerular diseases associated with nephrotic syndrome. J Am Soc Nephrol 2006;17:3139-48.
30. Martin H. Laboratory measurement of urine albumin and urine total protein in screening for proteinuria in chronic kidney disease. Clin Biochem Rev 2011;32:97-102.

31. Miller WG, Bruns DE, Hortin GL, Sandberg S, Aakre KM, McQueen MJ, et al. Current issues in measurement and reporting of urinary albumin excretion. Clin Chem 2009;55:24-38. 